\title{
Semiclassical Universality of Parametric Spectral Correlations
}

\author{
Jack Kuipers ${ }^{1}$ and Martin Sieber ${ }^{2}$ \\ School of Mathematics, University of Bristol, Bristol BS8 1TW, UK
}

\begin{abstract}
We consider quantum systems with a chaotic classical limit that depend on an external parameter, and study correlations between the spectra at different parameter values. In particular, we consider the parametric spectral form factor $K(\tau, x)$ which depends on a scaled parameter difference $x$. For parameter variations that do not change the symmetry of the system we show by using semiclassical periodic orbit expansions that the small $\tau$ expansion of the form factor agrees with Random Matrix Theory for systems with and without time reversal symmetry.
\end{abstract}

PACS numbers:

03.65.Sq Semiclassical theories and applications.

05.45.Mt Semiclassical chaos ("quantum chaos").

\footnotetext{
${ }^{1}$ E-mail: jack.kuipers@bristol.ac.uk

${ }^{2}$ E-mail: m.sieber@bristol.ac.uk
} 


\section{Introduction}

One of the characteristic features of quantum systems with underlying chaotic dynamics lies in statistical fluctuations of their spectra. If the energy levels are scaled such that their mean separation is one then the statistical distribution of the levels of individual quantum chaotic systems are found to be universal in the semiclassical limit $\hbar \rightarrow 0$ and to agree with those of eigenvalues of random matrices [1]. The appropriate ensembles of random matrices depend only on symmetries of the system. Systems with or without time reversal symmetry (TRS) are described by the Gaussian Orthogonal Ensemble (GOE) and the Gaussian Unitary Ensemble (GUE) (in the absence of half-integer spin and other symmetries, which we will assume in the following).

Universality can be observed, however, not only in the spectrum of an individual quantum system, but also in the way in which the spectrum changes due to an external perturbation. In the following we consider quantum systems that depend on an external parameter whose alteration does not change the symmetry of the system and for which the classical dynamics is chaotic for any parameter value. Correlations between the spectra of these systems at different parameter values are also found to be universal functions of the parameter difference provided that the parameter is scaled in an appropriate way $[2,3]$. The universal correlation functions again agree with those of Random Matrix Theory (RMT) and have been derived for the GOE and the GUE. The early developments of parametric spectral correlations are reviewed in [4].

One main approach to understanding the connection between quantum chaos and RMT has been the application of semiclassical methods. One convenient quantity to characterize correlations within the spectrum of an individual quantum system is the spectral form factor $K(\tau)$, the Fourier transform of the two-point correlation function of the density of states. Within the semiclassical approximation it is expressed by a double sum over the periodic orbits of the classical system. An evaluation of this double sum in the diagonal approximation, which pairs orbits with themselves or their time reverse, yields the first term in the small $\tau$ expansion of $K(\tau)$ in agreement with RMT $[5,6]$. Higher order terms are due to pairs of correlated periodic orbits. The origin of these correlations was identified and the next-order term obtained for uniformly hyperbolic systems in [7,8]. The contributions of all correlated periodic orbit pairs that contribute to the small $\tau$ expansion of $K(\tau)$ were evaluated and summed up by combinatorial methods in $[9,10,11]$. Similar methods have been applied since to derive offdiagonal terms, for example, for the conductance $[12,13]$, the shot-noise $[14]$ and the GOE-GUE transition [15].

For parametric spectral correlation functions agreement with RMT has been obtained within the diagonal approximation $[16,17,18]$. In this article we go beyond the diagonal approximation and derive off-diagonal terms for the parametric spectral form factor. For systems without time reversal symmetry we derive all terms in the small $\tau$-expansion in a closed form. In the GOE case the method allows the calculation of arbitrarily many terms in the expansion. We compare the expansion up to $\tau^{7}$ with the result of RMT and find exact agreement. One main reason for the universal result is that in the semiclassical limit $\hbar \rightarrow 0$ the relevant quantum fluctuations are due to very small parameter variations on the classical scale. One assumption is that the parameter dependence is in some sense typical. Specifically, we assume that the derivatives of the actions of very long periodic orbits with respect to the parameter have a Gaussian distribution $[17,18]$. This excludes, for example, rank-one perturbations for which off-diagonal terms were calculated in [19].

In section 2 we introduce the parametric spectral form factor and in section 3 we state results 
of Random Matrix Theory for it. In section 4 we consider its semiclassical approximation and the diagonal approximation, while in section 5 we derive the off-diagonal terms. For systems without time reversal symmetry the expansion is summed in section 6 , and section 7 contains our conclusions.

While writing up our paper the preprint [20], which is closely related to our work, appeared on the archive. Nagao et al. investigate parametric correlations that depend on a magnetic field difference, and obtain the universal results for the GUE case and the GOE-GUE transition by periodic orbit expansions. Our work is complementary in that we treat arbitrary parameters and consider also the GOE case.

\section{The parametric spectral form factor}

One way to characterize fluctuations in quantum spectra is to consider correlation functions of the density of states. For parametric correlations the density of states depends on the energy $E$ as well as on a parameter $X$, and in the semiclassical regime it can be written as the sum of a mean part and an oscillatory part

$$
d(E, X)=\sum_{n} \delta\left(E-E_{n}(X)\right) \approx \bar{d}(E, X)+d^{\mathrm{osc}}(E, X)
$$

where $E_{n}(X), n=1,2, \ldots$, is the $n$-th energy level as a function of the parameter $X$. The mean density of states in an $f$-dimensional system is given by $\bar{d}(E, X) \sim \Omega(E, X) /(2 \pi \hbar)^{f}$ in the semiclassical limit $\hbar \rightarrow 0 . \Omega(E, X)$ is the volume of the surface of constant energy in phase space at energy $E$ and parameter $X$.

In order to obtain a universal parametric spectral correlation function one has to perform two unfoldings, one in energy $E$ and one in the parameter $X$ of the system. A new energy parameter is defined by

$$
\tilde{E}=\bar{N}(E, X)
$$

where $\bar{N}(E, X)$ is the mean part of the spectral staircase $N(E, X)=\int_{-\infty}^{E} \mathrm{~d} E^{\prime} d\left(E^{\prime}, X\right)$. In terms of the new energy $\tilde{E}$ the density of states has a mean value of one. The spectral statistics are evaluated in the semiclassical limit in an interval $\Delta \tilde{E}$ that is classically small but contains a large number of energy levels, i.e. it satisfies $\tilde{E} \gg \Delta \tilde{E} \gg 1$.

A new parameter $\tilde{X}$ is introduced by $[2,21]$

$$
\tilde{X}=\int_{X_{0}}^{X} d X^{\prime} \sigma\left(X^{\prime}\right), \quad \sigma\left(X^{\prime}\right)=\sqrt{\left\langle v_{n}\left(X^{\prime}\right)^{2}\right\rangle},
$$

where $v_{n}(X)=\partial \tilde{E}_{n} / \partial X$ are the level velocities and the average is performed over the levels in the interval $\Delta \tilde{E} . X_{0}$ is an arbitrary parameter value at which $\tilde{X}=0$. In terms of the new parameter $\tilde{X}$ the level velocities have a unit variance.

We may then define the universal two-point correlation function by

$$
R_{2}(\eta, x)=\left\langle\tilde{d}^{\text {osc }}\left(\tilde{E}+\frac{\eta}{2}, \tilde{X}+\frac{x}{2}\right) \tilde{d}^{\text {osc }}\left(\tilde{E}-\frac{\eta}{2}, \tilde{X}-\frac{x}{2}\right)\right\rangle_{\tilde{E}, \tilde{X}},
$$

where $\tilde{d}(\tilde{E}, \tilde{X})$ is the density of states of the unfolded spectrum, and the average is performed over the energy interval $\Delta \tilde{E}$ as well as over a parameter interval $\Delta \tilde{X}$. The relation to the 
original density of states is given by

$$
\tilde{d}(\tilde{E}, \tilde{X})=\frac{\partial N(E, X)}{\partial E} \frac{\partial E}{\partial \tilde{E}}=\frac{d(E, X)}{\bar{d}(E, X)},
$$

and in the semiclassical limit we find that

$$
R_{2}(\eta, x) \sim \frac{\left\langle d^{\text {osc }}\left(E+\frac{\eta}{2 d}+\frac{x \rho}{2 \sigma}, X+\frac{x}{2 \sigma}\right) d^{\text {osc }}\left(E-\frac{\eta}{2 d}-\frac{x \rho}{2 \sigma}, X-\frac{x}{2 \sigma}\right)\right\rangle_{E, X}}{\bar{d}(E, X)^{2}} .
$$

Equation (6) has been obtained by linearizing the unfolding equations (2) and (3), because $x$ and $\eta$ correspond to small changes on the classical scale, since $\bar{d}$ is of the order $\hbar^{-f}$ and $\sigma=\sigma(X)$ is of the order of $\hbar^{-(f+1) / 2}$ (see equation (25) later). The term $x / 2 \rho \sigma$ takes account of the change of the energy when $X$ is changed while keeping $\tilde{E}$ fixed

$$
\rho=\left.\frac{\partial E}{\partial X}\right|_{\tilde{E}}=-\frac{\partial \bar{N} / \partial X}{\partial \bar{N} / \partial E} .
$$

In the following, we will consider the parametric spectral form factor which is obtained by a Fourier transform of the parametric two-point correlation function

$$
K(\tau, x)=\int_{-\infty}^{\infty} R_{2}(\eta, x) e^{-2 \pi i \eta \tau} d \eta
$$

\section{Results from Random Matrix Theory}

The parametric two-point correlation function $R_{2}(\eta, x)$ has been derived in the context of disordered systems $[2,22]$ for the GUE and the GOE. For the GUE case it is given by

$$
R_{2}^{\mathrm{GUE}}(\eta, x)=\frac{1}{2} \int_{-1}^{1} \mathrm{~d} \lambda \int_{1}^{\infty} \mathrm{d} \lambda_{1} \cos \left(\pi \omega\left(\lambda_{1}-\lambda\right)\right) \mathrm{e}^{-\pi^{2} x^{2}\left(\lambda_{1}^{2}-\lambda^{2}\right) / 2}
$$

After performing the Fourier transform in (8) to obtain the parametric form factor we arrive at

$$
K^{\mathrm{GUE}}(\tau, x)=\frac{1}{2} \int_{-1}^{1} \mathrm{~d} \lambda \int_{1}^{\infty} \mathrm{d} \lambda_{1} \mathrm{e}^{-\pi^{2} x^{2}\left(\lambda_{1}^{2}-\lambda^{2}\right) / 2}\left[\delta\left(\lambda_{1}-\lambda-2 \tau\right)+\delta\left(\lambda_{1}-\lambda+2 \tau\right)\right]
$$

Because $\tau$ is positive and $\lambda_{1} \geq \lambda$ the second delta function does not contribute. From the first delta function we get the relation $2 \tau=\lambda_{1}-\lambda$. In the case $\tau<1$, which we consider in the following, the domain of integration for $\lambda_{1}$ is reduced to $1 \leq \lambda_{1} \leq 1+2 \tau$, and we obtain

$$
K^{\mathrm{GUE}}(\tau, x)=\frac{1}{2} \int_{1}^{1+2 \tau} \mathrm{d} \lambda_{1} \mathrm{e}^{2 \pi^{2} x^{2} \tau\left(\tau-\lambda_{1}\right)}=\frac{\sinh \left(2 \pi^{2} x^{2} \tau^{2}\right)}{2 \pi^{2} x^{2} \tau} \mathrm{e}^{-2 \pi^{2} x^{2} \tau}, \quad \tau<1 .
$$

For comparison with the semiclassical expansion we expand the sinh-function and define $B=$ $2 \pi^{2} x^{2} / \kappa$ where $\kappa=1$ and 2 for the GUE and GOE cases, respectively.

$$
K^{\mathrm{GUE}}(\tau, x)=\mathrm{e}^{-B \tau} \sum_{n=0}^{\infty} \frac{B^{2 n} \tau^{4 n+1}}{(2 n+1) !}
$$


The parametric correlation function for the GOE case is given by a triple integral

$$
\begin{aligned}
R_{2}^{\mathrm{GOE}}(\eta, x)=\int_{-1}^{1} \mathrm{~d} \lambda & \int_{1}^{\infty} \mathrm{d} \lambda_{1} \int_{1}^{\infty} \mathrm{d} \lambda_{2} \cos \left(\pi \omega\left(\lambda-\lambda_{1} \lambda_{2}\right)\right) \frac{\left(1-\lambda^{2}\right)\left(\lambda-\lambda_{1} \lambda_{2}\right)^{2}}{\left(2 \lambda \lambda_{1} \lambda_{2}-\lambda^{2}-\lambda_{1}{ }^{2}-\lambda_{2}{ }^{2}+1\right)^{2}} \\
& \times \mathrm{e}^{-\pi^{2} x^{2}\left(2 \lambda_{1}{ }^{2} \lambda_{2}{ }^{2}-\lambda^{2}-\lambda_{1}{ }^{2}-\lambda_{2}{ }^{2}+1\right) / 4}
\end{aligned}
$$

Evaluating the Fourier transform to obtain the parametric form factor results in a sum of two delta-functions

$$
\begin{aligned}
K^{\mathrm{GOE}}(\tau, x)= & \int_{-1}^{1} \mathrm{~d} \lambda \int_{1}^{\infty} \mathrm{d} \lambda_{1} \int_{1}^{\infty} \mathrm{d} \lambda_{2} \frac{\left(1-\lambda^{2}\right)\left(\lambda-\lambda_{1} \lambda_{2}\right)^{2}}{\left(2 \lambda \lambda_{1} \lambda_{2}-\lambda^{2}-\lambda_{1}{ }^{2}-\lambda_{2}{ }^{2}+1\right)^{2}} \\
& \times \mathrm{e}^{-\pi^{2} x^{2}\left(2 \lambda_{1}{ }^{2} \lambda_{2}{ }^{2}-\lambda^{2}-\lambda_{1}{ }^{2}-\lambda_{2}{ }^{2}+1\right) / 4}\left[\delta\left(\lambda-\lambda_{1} \lambda_{2}-2 \tau\right)+\delta\left(\lambda-\lambda_{1} \lambda_{2}+2 \tau\right)\right] .
\end{aligned}
$$

Because $\tau$ is positive and $\lambda_{1} \lambda_{2} \geq \lambda$ only the second delta function contributes, giving the relation $\lambda=\lambda_{1} \lambda_{2}-2 \tau$. As we are again considering the case when $\tau<1$ our domain of integration for the other two variables is given by $1 \leq \lambda_{1} \leq 1+2 \tau$ and $1 \leq \lambda_{2} \leq \frac{1+2 \tau}{\lambda_{1}}$. When we perform the integral over $\lambda$ we are left with

$$
\begin{gathered}
K^{\mathrm{GOE}}(\tau, x)=\int_{1}^{1+2 \tau} \mathrm{d} \lambda_{1} \int_{1}^{\frac{1+2 \tau}{\lambda_{1}}} \mathrm{~d} \lambda_{2} \frac{4 \tau^{2}\left(1-\lambda_{1}{ }^{2} \lambda_{2}{ }^{2}+4 \tau \lambda_{1} \lambda_{2}-4 \tau^{2}\right)}{\left(1+\lambda_{1}{ }^{2} \lambda_{2}{ }^{2}-\lambda_{1}{ }^{2}-\lambda_{2}{ }^{2}-4 \tau^{2}\right)^{2}} \\
\times \mathrm{e}^{-\pi^{2} x^{2}\left(1+\lambda_{1}{ }^{2} \lambda_{2}{ }^{2}-\lambda_{1}{ }^{2}-\lambda_{2}{ }^{2}+4 \tau \lambda_{1} \lambda_{2}-4 \tau^{2}\right) / 4} .
\end{gathered}
$$

In order to evaluate this integral as a series in $\tau$ it is useful to remove the $\tau$ dependence from the limits. This is done by changing the integration variables using $\lambda_{1}=1+\tau y_{1}$ and $\lambda_{1} \lambda_{2}=1+\tau y_{2}$. Then the expansion of the parametric form factor is obtained by expanding the integrand for small values of $\tau$.

$$
\begin{gathered}
K^{\mathrm{GOE}}(\tau, x)=\int_{0}^{2} \mathrm{~d} y_{1} \int_{y_{1}}^{2} \mathrm{~d} y_{2}\left\{\frac{2-y_{2}}{2\left(1-y_{1} y_{2}+y_{1}^{2}\right)^{2}} \tau+\left[\frac{y_{1}\left(y_{2}-2\right)\left(4-y_{1} y_{2}+2 y_{1}^{2}-y_{2}^{2}\right)}{2\left(1-y_{1} y_{2}+y_{1}^{2}\right)^{3}}\right.\right. \\
\left.\left.+\frac{\left(2-y_{2}\right)\left(2+2 \pi^{2} x^{2}-6 y_{1}-y_{2}\right)}{4\left(1-y_{1} y_{2}+y_{1}^{2}\right)^{2}}\right] \tau^{2}+\ldots\right\} .
\end{gathered}
$$

Using Maple we performed this expansion up to seventh order and evaluated the integrals with the following result

$$
\begin{aligned}
K^{\mathrm{GOE}}(\tau, x) & =2 \tau-\left(2 \pi^{2} x^{2}+2\right) \tau^{2}+\left(\pi^{4} x^{4}+2\right) \tau^{3}-\left(\frac{\pi^{6} x^{6}}{3}-\pi^{4} x^{4}+\frac{8}{3}\right) \tau^{4} \\
& +\left(\frac{\pi^{8} x^{8}}{12}-\frac{2 \pi^{6} x^{6}}{3}+\frac{2 \pi^{4} x^{4}}{3}+4\right) \tau^{5} \\
& -\left(\frac{\pi^{10} x^{10}}{60}-\frac{\pi^{8} x^{8}}{4}+\pi^{6} x^{6}+\frac{\pi^{4} x^{4}}{3}+\frac{32}{5}\right) \tau^{6} \\
& +\left(\frac{\pi^{12} x^{12}}{360}-\frac{\pi^{10} x^{10}}{15}+\frac{7 \pi^{8} x^{8}}{12}-\frac{2 \pi^{6} x^{6}}{15}+\frac{\pi^{4} x^{4}}{5}+\frac{32}{3}\right) \tau^{7} .
\end{aligned}
$$

For comparison with the semiclassical result it is convenient to extract an exponential factor 
from this expansion.

$$
\begin{aligned}
K^{\mathrm{GOE}}(\tau, x) & =\mathrm{e}^{-B \tau}\left[2 \tau-2 \tau^{2}-(2 B-2) \tau^{3}+\left(2 B-\frac{8}{3}\right) \tau^{4}\right. \\
& +\left(\frac{5 B^{2}}{3}-\frac{8 B}{3}+4\right) \tau^{5}-\left(\frac{5 B^{2}}{3}-4 B+\frac{32}{5}\right) \tau^{6} \\
& \left.-\left(\frac{41 B^{3}}{45}-\frac{11 B^{2}}{5}+\frac{32 B}{5}-\frac{32}{3}\right) \tau^{7}+\ldots\right],
\end{aligned}
$$

where $B$ has been defined after equation (11).

\section{Semiclassical approximation}

In this section we derive a semiclassical expression for the parametric spectral form factor. It is closely related to semiclassical approximations for the parametric two-point correlation function of the density of states $[17,18]$. We start by expressing the density of states with the Gutzwiller trace formula [23]

$$
d^{\mathrm{osc}}(E, X) \approx \frac{1}{\pi \hbar} \operatorname{Re} \sum_{\gamma} A_{\gamma} \exp \left(\frac{\mathrm{i}}{\hbar} S_{\gamma}\right), \quad \text { where } \quad A_{\gamma}=\frac{T_{\gamma}}{R_{\gamma} \sqrt{\left|\operatorname{det}\left(M_{\gamma}-1\right)\right|}} \mathrm{e}^{-\mathrm{i} \pi \mu_{\gamma} / 2}
$$

The sum runs over all periodic orbits of the system with period $T_{\gamma}$, repetition number $R_{\gamma}$, stability matrix $M_{\gamma}$ and Maslov index $\mu_{\gamma}$.

The action is expanded in first order in the energy difference and the parameter difference

$$
S_{\gamma}\left(E \pm \frac{\eta}{2 \bar{d}} \pm \frac{x \rho}{2 \sigma}, X \pm \frac{x}{2 \sigma}\right) \approx S_{\gamma}(E, X) \pm T_{\gamma}(E, X) \frac{\eta}{2 \bar{d}} \pm Q_{\gamma}(E, X) \frac{x}{2 \sigma},
$$

where

$$
Q_{\gamma}=\left.\frac{\partial S_{\gamma}}{\partial X}\right|_{\tilde{E}}=\rho \frac{\partial S_{\gamma}}{\partial E}+\frac{\partial S_{\gamma}}{\partial X}
$$

is the parametric velocity.

After inserting (19) and (20) into (6) and (8) and evaluating the integral to leading semiclassical order, we arrive at

$$
K(\tau, x)=\frac{1}{T_{H}}\left\langle\sum_{\gamma, \gamma^{\prime}} A_{\gamma} A_{\gamma^{\prime}}^{*} \mathrm{e}^{\mathrm{i}\left(S_{\gamma}-S_{\gamma^{\prime}}\right)} \mathrm{e}^{\frac{\mathrm{i} x\left(Q_{\gamma}+Q_{\gamma^{\prime}}\right)}{2 \sigma \hbar}} \delta\left(T-\frac{T_{\gamma}+T_{\gamma^{\prime}}}{2}\right)\right\rangle
$$

where $T_{H}=2 \pi \hbar \bar{d}(E)$ is the Heisenberg time, and $\tau=T / T_{H}$. Terms which have a sum of the actions in the exponent have been neglected, because they average away. The semiclassical expression (22) is the quantity that we will evaluate in the following.

The diagonal approximation involves pairs of orbits that are either identical or related by time reversal. It has the form

$$
K(\tau, x)=\frac{\kappa}{T_{H}}\left\langle\sum_{\gamma}\left|A_{\gamma}\right|^{2} \mathrm{e}^{\frac{\mathrm{i} x Q \gamma}{\sigma \hbar}} \delta\left(T-T_{\gamma}\right)\right\rangle
$$

where $\kappa$ is 2 if the system has time reversal symmetry and 1 if it does not. 
One main ingredient in the following semiclassical calculation is the distribution of the parametric velocities $Q_{\gamma}$ in the limit of very long periodic orbits. It has been shown that the $Q_{\gamma}$ have a mean value of zero and a variance proportional to their period [16]

$$
\left\langle Q_{\gamma}\right\rangle=0, \quad\left\langle Q_{\gamma}^{2}\right\rangle \sim a T, \quad T \rightarrow \infty,
$$

where the averages are performed over trajectories with period around $T$. It has been motivated that the $Q_{\gamma}$ have a Gaussian distribution for long periodic orbits (see [18]) and this is the main assumption that we will use in the following. The proportionality factor $a$ in (24) is related to the variance of the level velocities. [24, 21]

$$
\sigma^{2} \sim \frac{a \kappa \bar{d}}{2 \pi \hbar} .
$$

Using the Gaussian assumption for the distribution of the parametric velocities and (25) we perform the average over the $Q_{\gamma}$, assuming that it can be done independently, and obtain

$$
\left\langle\mathrm{e}^{\frac{\mathrm{i} x Q_{\gamma}}{\sigma \hbar}}\right\rangle=\mathrm{e}^{-\frac{x^{2} a T}{2 \sigma^{2} \hbar^{2}}}=\mathrm{e}^{-B T / T_{H}},
$$

where $B=2 \pi^{2} x^{2} / \kappa$ is the same quantity as defined after equation (11). The remaining sum over periodic orbits can be evaluated with the Hannay-Ozorio de Almeida sum rule [5]

$$
\sum_{\gamma}\left|A_{\gamma}\right|^{2} \delta\left(T-T_{\gamma}\right) \approx T .
$$

We find that the diagonal approximation is given by

$$
K(\tau)=\kappa \tau \mathrm{e}^{-B \tau}
$$

in agreement with the first term in the expansion of the random matrix results, (12) and (18).

\section{Off-diagonal contributions}

The off-diagonal terms of the parametric form factor are due to pairs of trajectories which are correlated $[7,10]$. In the following we briefly review the main steps in the derivation of the semiclassical expansion of the spectral form factor (in our notation $K(\tau, x=0)$ ) according to $[10,11]$. The correlations that are important for the expansion of the form factor for small $\tau$ are due to close self-encounters of a periodic orbit in which two or more stretches of an orbit are almost identical, possibly up to time reversal. In general, a long periodic orbit has many of these encounter regions, and they are connected by long parts of the orbit, the so-called "loops". The correlated pairs of orbits are almost identical along the loops, but they differ in the way in which the loops are connected in the encounter regions. Correlated orbit pairs have certain "structures" that are characterized by the number of encounter regions $V$ in which the loops are connected in a different way, the number of involved orbit stretches $l_{\alpha}$ in each encounter region $\alpha$, and the way in which the loops are connected by these stretches. A more accurate definition of structures can be given by putting them in a one-to-one relation with permutation matrices that describe the reconnections of the loops. One defines further a vector 
$\boldsymbol{v}$ whose $l$-th component, $v_{l}$, specifies the number of encounter regions with $l$ stretches, and the total number of orbit stretches is denoted by $L$. Hence

$$
V=\sum_{l \geq 2} v_{l}, \quad L=\sum_{\alpha} l_{\alpha}=\sum_{l \geq 2} l v_{l}
$$

The semiclassical contribution to the form factor is evaluated in two steps. First the summation over orbit pairs with the same structure is evaluated by using that long periodic orbits are uniformly distributed over the surface of constant energy in phase space. Then the summation over the different structures is performed which is a combinatorial problem.

In the following we present some details of this calculation. In each encounter region $\alpha$ with $l_{\alpha}$ orbit stretches one chooses a perpendicular Poincaré surface that is centered on one of the stretches. The relative positions of the piercings of the other stretches through the Poincaré surface are described by coordinates along the stable and unstable manifolds. The partner periodic orbit connects the loops that start and end at the encounter region in a different way and the resulting contribution to the action difference is given in the linearized approximation by

$$
(\Delta S)_{\alpha}=\sum_{j=1}^{l_{\alpha}-1} s_{\alpha j} u_{\alpha j}
$$

where $s_{\alpha j}, u_{\alpha j}, j=1, \ldots, l_{\alpha}-1$ are appropriate differences of the coordinates along the stable and unstable manifolds. For ease of notation we discuss here the two-dimensional case in which the coordinates $s_{\alpha j}$ and $u_{\alpha j}$ are scalars. If the Poincaré surface is moved along the stretches in the encounter region these coordinates decrease or increase, exponentially, however their product remains constant. The length of the encounter region is determined by requiring that all coordinates remain smaller than an arbitrary small constant $c$ whose exact size is not relevant for the following calculations.

The uniform distribution of the long periodic orbits on the energy shell is then invoked to sum over all orbit pairs with the same structure. It is convenient to also sum over all structures with the same vector $\boldsymbol{v}$ and express it in the form

$$
K_{\boldsymbol{v}}(\tau)=\frac{1}{T_{H}} \sum_{\left(\gamma, \gamma^{\prime}\right)}^{\text {fixed } \boldsymbol{v}}\left|A_{\gamma}\right|^{2} e^{\mathrm{i} \Delta S_{\gamma} / \hbar} \delta\left(T-T_{\gamma}\right)=N(\boldsymbol{v}) \kappa \tau \int d^{L-V} s d^{L-V} u \frac{w_{T}(\boldsymbol{s}, \boldsymbol{u})}{L} \mathrm{e}^{\mathrm{i} \boldsymbol{s} \boldsymbol{u} / \hbar}
$$

so that $K(\tau)=\kappa \tau+\sum_{\boldsymbol{v}} K_{\boldsymbol{v}}(\tau) . N(\boldsymbol{v})$ is the number of structures with the same $\boldsymbol{v}$, and $\boldsymbol{s}$ and $\boldsymbol{u}$ are vectors whose components are the $s_{\alpha j}$ and $u_{\alpha j}$ for all $\alpha$ and $j$. In (31) the amplitudes and periods of the two correlated orbits are set equal. $w_{T}(\boldsymbol{s}, \boldsymbol{u})$ is the density of the selfencounters for a given structure and separation coordinates $s_{\alpha j}$ and $u_{\alpha j}$. For long orbits it is given asymptotically by

$$
\frac{w_{T}(\boldsymbol{s}, \boldsymbol{u})}{L}=\frac{T\left(T-\sum_{\alpha} l_{\alpha} t_{\mathrm{enc}}^{\alpha}\right)^{L-1}}{L ! \Omega^{L-V} \prod_{\alpha} t_{\mathrm{enc}}^{\alpha}} .
$$

The factor $1 / L$ in (31) takes care of an overcounting related to the choice of an initial point of the trajectory $[10]$.

The integral in (31) is evaluated by using

$$
\int \prod_{j} \mathrm{~d} s_{\alpha j} \mathrm{~d} u_{\alpha j}\left(t_{\mathrm{enc}}^{\alpha}\right)^{k} \mathrm{e}^{\mathrm{i} \sum_{j} s_{\alpha j} u_{\alpha j} / \hbar} \approx\left\{\begin{array}{ll}
0 & \text { if } k=-1 \text { or } k \geq 1 \\
(2 \pi \hbar)^{l_{\alpha}-1} & \text { if } k=0
\end{array} .\right.
$$


Hence after expanding the numerator of (32) the only term that survives is the one that contains a product of all encounter times $t_{\text {enc }}^{\alpha}$ which is cancelled by the denominator. Therefore one can replace the density $w_{T}$ in the integral by

$$
\frac{w_{T}(\boldsymbol{s}, \boldsymbol{u})}{L} \Longrightarrow \frac{T}{L ! \Omega^{L-V}} \frac{(L-1) ! T^{L-V-1}(-1)^{V} \prod_{l} l^{v_{l}}}{(L-V-1) !}
$$

where we used that $\prod_{\alpha} l_{\alpha}=\prod_{l} l^{v_{l}}$. Inserting (34) into (31) and evaluating the integral with the use of (33) yields the contribution from orbit pairs with fixed $\boldsymbol{v}$,

$$
K_{\boldsymbol{v}}(\tau)=\kappa \tau^{L-V+1} N(\boldsymbol{v}) \frac{(-1)^{V} \prod_{l} l^{v_{l}}}{L(L-V-1) !}
$$

As can be seen, vectors $\boldsymbol{v}$ with the same value of $L-V+1$ contribute to the same power of $\tau$. Hence the power series expansion of the form factor can be obtained by summing over all $\boldsymbol{v}$ with the same $L-V+1$.

$$
K(\tau)=\kappa \tau+\sum_{n=2}^{\infty} K_{n} \tau^{n}
$$

where

$$
K_{n}=\frac{\kappa}{(n-2) !} \sum_{\boldsymbol{v}}^{L-V+1=n} \tilde{N}(\boldsymbol{v}), \quad \tilde{N}(\boldsymbol{v})=\left(N(\boldsymbol{v})(-1)^{V} \prod_{l} l^{v_{l}}\right) / L
$$

By deriving a recurrence relation for the numbers $\tilde{N}(\boldsymbol{v})$ it is shown that the semiclassical expansion in (36) agrees with the small $\tau$ expansion of the form factor of RMT in cases with or without time reversal symmetry $[9,10,11]$.

Let us now come back to the parametric form factor $K(\tau, x)$. We have to evaluate

$$
K(\tau, x)=\frac{1}{T_{H}}\left\langle\sum_{\gamma, \gamma^{\prime}}\left|A_{\gamma}\right|^{2} \mathrm{e}^{\frac{\mathrm{i}\left(S_{\gamma}-S_{\gamma^{\prime}}\right)}{\hbar}} \mathrm{e}^{\frac{\mathrm{i} x Q_{\gamma}}{\sigma \hbar}} \delta\left(T-T_{\gamma}\right)\right\rangle .
$$

It now contains an additional term involving the parametric velocities $Q_{\gamma}$. As for the diagonal approximation we assume that the average over the $Q_{\gamma}$ can be performed independently from the actions and amplitudes of the orbits. However, we have to look at the average (26) more carefully. It is valid as long as there are no systematic correlations between different parts of the same periodic orbit. Long periodic orbits have many encounter regions in which different stretches of an orbit are almost identical and cannot be considered as independent or uncorrelated. When we calculate the contribution to the form factor of orbit pairs with a certain structure, then we average over periodic orbits that all have the same number and types of encounter regions. The corresponding correlations between different parts of an orbit have to be taken into account. We should consider the average over the parametric velocities for the loops and encounter regions separately. The contribution from a loop is

$$
\left\langle\mathrm{e}^{\frac{\mathrm{i} x Q_{\gamma}^{\text {loop }}}{\sigma \hbar}}\right\rangle=\mathrm{e}^{-B T_{\text {loop }} / T_{H}}
$$

while the contribution from the $l$ orbit stretches in an $l$-encounter region is

$$
\left\langle\mathrm{e}^{\frac{\mathrm{i} x l Q_{\gamma}^{e n c}}{\sigma \hbar}}\right\rangle=\mathrm{e}^{-B l^{2} t_{e n c} / T_{H}} .
$$


This means that the average over the parametric velocities is now given by

$$
\left\langle\mathrm{e}^{\frac{\mathrm{i} x Q_{\gamma}}{\sigma \hbar}}\right\rangle=\mathrm{e}^{-B\left(T-\sum_{\alpha} l_{\alpha} t_{\mathrm{enc}}^{\alpha}\right) / T_{H}} \mathrm{e}^{-B \sum_{\alpha} l_{\alpha}^{2} t_{\mathrm{enc}}^{\alpha} / T_{H}}
$$

The contribution of all orbit pairs with encounter regions described by $\boldsymbol{v}$ then have the form

$$
K_{\boldsymbol{v}}(\tau, x)=\frac{1}{T_{H}} \sum_{\left(\gamma, \gamma^{\prime}\right)}^{\text {fixed } \boldsymbol{v}}\left|A_{\gamma}\right|^{2} e^{\mathrm{i} \Delta S_{\gamma} / \hbar} \mathrm{e}^{\frac{\mathrm{i} x Q_{\gamma}}{\sigma \hbar}} \delta\left(T-T_{\gamma}\right)=N(\boldsymbol{v}) \kappa \tau \int d^{L-V} s d^{L-V} u \frac{z_{T}(\boldsymbol{s}, \boldsymbol{u})}{L} \mathrm{e}^{\mathrm{i} \boldsymbol{s} \boldsymbol{u} / \hbar},
$$

where

$$
\frac{z_{T}(s, u)}{L}=\frac{w_{T}(s, u)}{L}\left\langle\mathrm{e}^{\frac{\mathrm{i} x Q_{\gamma}}{\sigma \hbar}}\right\rangle=\frac{\mathrm{e}^{-B T / T_{H}} T\left(T-\sum_{\alpha} l_{\alpha} t_{e n c}^{\alpha}\right)^{L-1} \prod_{\alpha} \mathrm{e}^{-B l_{\alpha}\left(l_{\alpha}-1\right) t_{e n c}^{\alpha} / T_{H}}}{L ! \Omega^{L-V} \prod_{\alpha} t_{e n c}^{\alpha}} .
$$

Here $\alpha$ labels the $V$ different encounters, each being a $l_{\alpha}$-encounter. Again because of (33) the only terms that contribute in the semiclassical limit are those where the encounter times in the numerator and denominator cancel exactly. As a first step we can expand the exponentials as a power series up to first order

$$
\frac{z_{T}(s, u)}{L}=\frac{\mathrm{e}^{-B T / T_{H}} T\left(T-\sum_{\alpha} l_{\alpha} t_{e n c}^{\alpha}\right)^{L-1} \prod_{\alpha}\left(1-l_{\alpha}\left(l_{\alpha}-1\right) B t_{e n c}^{\alpha} / T_{H}\right)}{L ! \Omega^{L-V} \prod_{\alpha} t_{e n c}^{\alpha}} .
$$

To obtain a product of the $V$ different encounter times in the numerator we can take $r$ of them from the product over $\alpha$ and $V-r$ of them from the bracket with the exponent $L-1$. The corresponding coefficient is obtained by combinatorial considerations. Then we sum over all values of $r$ from 0 to $V$, and the result is

$$
\frac{z_{T}(s, u)}{L} \Longrightarrow \frac{\mathrm{e}^{-B T / T_{H}} T}{L ! \Omega^{L-V}} \sum_{r=0}^{V} \frac{T^{L-V+r-1} B^{r}(L-1) !(-1)^{V} \prod l^{v_{l}}}{T_{H}^{r}(L-V-1+r) ! r !} \sum_{\substack{\alpha_{1}, \ldots, \alpha_{r} \\ \text { distinct }}}\left(l_{\alpha_{1}}-1\right) \times \ldots \times\left(l_{\alpha_{r}}-1\right)
$$

We insert this into (42), evaluate the integral with formula (33), and obtain

$$
K_{\boldsymbol{v}}(\tau, x)=\frac{\kappa N(\boldsymbol{v}) \frac{\mathrm{e}^{-B \tau}}{L} \sum_{r=0}^{V} \tau^{L-V+r+1} B^{r}(-1)^{V} \prod l^{v_{l}}}{(L-V-1+r) ! r !} \sum_{\substack{\alpha_{1}, \ldots, \alpha_{r} \\ \text { distinct }}}\left(l_{\alpha_{1}}-1\right) \times \ldots \times\left(l_{\alpha_{r}}-1\right) .
$$

The contribution of orbits for the different types of encounters can be calculated with this formula, and they are shown for orbit pairs with $L-V \leq 4$ in table 1 . The vectors $\boldsymbol{v}$ are represented in the form $(2)^{v_{2}}(3)^{v_{3}} \ldots$ and the horizontal lines separate vectors $\boldsymbol{v}$ with different value of $L-V$. The numbers $N(\boldsymbol{v})$ can be calculated by combinatorial methods [1]].

To find the total contribution to the form factor we now multiply the middle column that contains $K_{\boldsymbol{v}} /(\kappa N(\boldsymbol{v}))$ by $\kappa$ and $N(\boldsymbol{v})$, add the diagonal approximation and sum over different $\boldsymbol{v}$. If we do that for all orbits pairs with $L-V \leq 8$ for the case without time reversal symmetry $(\kappa=1)$, we obtain the expansion for the form factor in $\tau$ up to 9 th order

$$
K(\tau)=\mathrm{e}^{-B \tau}\left[\tau+\frac{B^{2} \tau^{5}}{6}+\frac{B^{4} \tau^{9}}{120}+\ldots\right] .
$$




\begin{tabular}{|c|c|c|c|c|c|}
\hline $\boldsymbol{v}$ & $L$ & $V$ & $K_{\boldsymbol{v}}(\tau, x) /(\kappa N(\boldsymbol{v}))$ & $N(\boldsymbol{v})$, no TRS & $N(\boldsymbol{v})$, TRS \\
\hline$(2)^{1}$ & 2 & 1 & $-\mathrm{e}^{-B \tau}\left(\tau^{2}+B \tau^{3}\right)$ & - & 1 \\
\hline$(2)^{2}$ & 4 & 2 & $\mathrm{e}^{-B \tau}\left(\tau^{3}+B \tau^{4}+\frac{B^{2} \tau^{5}}{6}\right)$ & 1 & 5 \\
$(3)^{1}$ & 3 & 1 & $-\mathrm{e}^{-B \tau}\left(\tau^{3}+B \tau^{4}\right)$ & 1 & 4 \\
\hline$(2)^{3}$ & 6 & 3 & $-\mathrm{e}^{-B \tau}\left(\frac{2 \tau^{4}}{3}+\frac{2 B \tau^{5}}{3}+\frac{B^{2} \tau^{6}}{6}+\frac{B^{3} \tau^{7}}{90}\right)$ & - & 60 \\
$(2)^{1}(3)^{1}$ & 5 & 2 & $\mathrm{e}^{-B \tau}\left(\frac{3 \tau^{4}}{5}+\frac{3 B \tau^{5}}{5}+\frac{B^{2} \tau^{6}}{10}\right)$ & - & 20 \\
$(4)^{1}$ & 4 & 1 & $-\mathrm{e}^{-B \tau}\left(\frac{\tau^{4}}{2}+\frac{B \tau^{5}}{2}\right)$ & - & 509 \\
\hline$(2)^{4}$ & 8 & 4 & $\mathrm{e}^{-B \tau}\left(\frac{\tau^{5}}{3}+\frac{B \tau^{6}}{3}+\frac{B^{2} \tau^{7}}{10}+\frac{B^{3} \tau^{8}}{90}+\frac{B^{4} \tau^{9}}{2520}\right)$ & 21 & 1092 \\
$(2)^{2}(3)^{1}$ & 7 & 3 & $-\mathrm{e}^{-B \tau}\left(\frac{2 \tau^{5}}{7}+\frac{2 B \tau^{6}}{7}+\frac{B^{2} \tau^{7}}{14}+\frac{B^{3} \tau^{8}}{210}\right)$ & 49 & 504 \\
$(2)^{1}(4)^{1}$ & 6 & 2 & $\mathrm{e}^{-B \tau}\left(\frac{2 \tau^{5}}{9}+\frac{2 B \tau^{6}}{9}+\frac{B^{2} \tau^{7}}{30}\right)$ & 24 & 228 \\
$(3)^{2}$ & 6 & 2 & $\mathrm{e}^{-B \tau}\left(\frac{\tau^{5}}{4}+\frac{B \tau^{6}}{4}+\frac{B^{2} \tau^{7}}{20}\right)$ & 12 & 148 \\
$(5)^{1}$ & 5 & 1 & $-\mathrm{e}^{-B \tau}\left(\frac{\tau^{5}}{6}+\frac{B \tau^{6}}{6}\right)$ & 8 & \\
\hline
\end{tabular}

Table 1: Contribution of different types of orbit pairs to the parametric form factor

This agrees with the first three terms of the expansion (12) in the section on RMT. It is noticable that when summing over terms with the same value of $L-V$, that all terms cancel apart from the highest order term from orbit pairs with only 2-encounters. In fact we will show this using a recurrence relation in appendix A. This allows us to calculate the expansion of the form factor to all orders in $\tau$ which is done in the next section.

For systems with time reversal symmetry $(\kappa=2)$ we sum over all contributions with $L-V \leq$ 6 , and obtain the expansion of the parametric form factor in $\tau$ up to 7 th order

$$
\begin{aligned}
K(\tau) & =\mathrm{e}^{-B \tau}\left[2 \tau-2 \tau^{2}-(2 B-2) \tau^{3}+\left(2 B-\frac{8}{3}\right) \tau^{4}\right. \\
& +\left(\frac{5 B^{2}}{3}-\frac{8 B}{3}+4\right) \tau^{5}-\left(\frac{5 B^{2}}{3}-4 B+\frac{32}{5}\right) \tau^{6} \\
& \left.-\left(\frac{41 B^{3}}{45}-\frac{11 B^{2}}{5}+\frac{32 B}{5}-\frac{32}{3}\right) \tau^{7}+\ldots\right]
\end{aligned}
$$

This agrees with the expansion of the RMT result (18).

\section{Systems without time reversal symmetry}

In this section we derive the full expansion of the parametric form factor for small $\tau$ for the case of systems without time reversal symmetry. For this purpose we rewrite the expansion $K(\tau, x)=\tau \mathrm{e}^{-B \tau}+\sum_{n=2}^{\infty} K_{\boldsymbol{v}}(\tau, x)$ with $K_{\boldsymbol{v}}(\tau, x)$ given in equation (46) in the following form

$$
K(\tau, x)=\tau e^{-B \tau}+e^{-B \tau} \sum_{n=2}^{\infty} \sum_{r=0}^{n-1} K_{n, r} \tau^{n+r} B^{r} .
$$


The coefficients $K_{n, r}$ have the form

$$
K_{n, r}=\frac{1}{(n-2) !} S_{n}\left[f_{r}(\boldsymbol{v})\right]
$$

where

$$
S_{n}\left[f_{r}(\boldsymbol{v})\right]=\sum_{\boldsymbol{v}}^{L-V+1=n} f_{r}(\boldsymbol{v}) \tilde{N}(\boldsymbol{v})
$$

and the functions $f_{r}(\boldsymbol{v})$ are given by

$$
f_{r}(\boldsymbol{v})=\frac{(L-V-1) !}{(L-V-1+r) ! r !} \sum_{\substack{\alpha_{1}, \ldots, \alpha_{r} \\ \text { distinct }}}\left(l_{\alpha_{1}}-1\right) \times \ldots \times\left(l_{\alpha_{r}}-1\right) .
$$

The first few functions are

$$
\begin{aligned}
& f_{0}(\boldsymbol{v})=1, \\
& f_{1}(\boldsymbol{v})=\frac{\sum_{\alpha}\left(l_{\alpha}-1\right)}{L-V}=\frac{\sum_{k} v_{k}(k-1)}{L-V}=1, \\
& f_{2}(\boldsymbol{v})=\frac{\sum_{\alpha_{1}, \alpha_{2}}\left(l_{\alpha_{1}}-1\right)\left(l_{\alpha_{2}}-1\right)-\sum_{\alpha}\left(l_{\alpha}-1\right)^{2}}{2(L-V)(L-V+1)}=\frac{(L-V)^{2}-\sum_{k} v_{k}(k-1)^{2}}{2(L-V)(L-V+1)} .
\end{aligned}
$$

We need to evaluate the quantities $S_{n}\left[f_{r}(\boldsymbol{v})\right]$ for $r<n$. In appendix A it is shown that $S_{n}\left[f_{r}(\boldsymbol{v})\right]=0$ for $r<n-1$. Hence the only non-vanishing terms in the expansion (49) are those with $r=n-1$.

$$
K(\tau, x)=\tau e^{-B \tau}+e^{-B \tau} \sum_{n=2}^{\infty} K_{n, n-1} \tau^{2 n-1} B^{n-1} .
$$

Since $r$ satisfies $r \leq V$ we have $V \geq n-1$. Together with the condition $L-V=n-1$ we find that $2 V \geq L$. This is only satisfied for orbit pairs with $V$ 2-encounters for which $\boldsymbol{v}=(2)^{V}$ and $L=2 \mathrm{~V}$. The contribution of these orbit pairs to the form factors can be calculated explicitly. We obtain from equations (50), (51) and (52) with $r=V=n-1, L=2 V$, and $l_{\alpha}=2$ for all $\alpha$

$$
K_{n, n-1}=\frac{\tilde{N}(\boldsymbol{v})}{(n-2) !} \frac{(L-V-1) !}{(L-V-1+r) !}=\frac{(-1)^{n-1} 2^{n-1}}{(2 n-2) !} N(\boldsymbol{v}) .
$$

The number $N(\boldsymbol{v})$ can be obtained from an explicit formula that has been derived for systems without time reversal symmetry in [25]. In our notation it has the form

$$
\mathcal{N}(\boldsymbol{v})=\frac{1}{L+1} \sum_{\boldsymbol{v}^{\prime} \leq \boldsymbol{v}} \frac{(-1)^{L^{\prime}-V^{\prime}} L^{\prime} !\left(L-L^{\prime}\right) !}{\prod_{k \geq 2} k^{v_{k}} v_{k}^{\prime} !\left(v_{k}-v_{k}^{\prime}\right) !} .
$$

The notation $\boldsymbol{v}^{\prime} \leq \boldsymbol{v}$ means that the sum runs over all integer vectors $\boldsymbol{v}^{\prime}$ whose components satisfy $0 \leq v_{k}^{\prime} \leq v_{k}$ for all $k$. Furthermore, $L^{\prime}=L\left(\boldsymbol{v}^{\prime}\right)$ and $V^{\prime}=V\left(\boldsymbol{v}^{\prime}\right)$. In the case of vectors $\boldsymbol{v}$ of the form $(2)^{V}$ the only non-vanishing component of $\boldsymbol{v}$ is $v_{2}=V$ and the sum runs over all vectors with component $v_{2}^{\prime}=m$ where $m=0, \ldots, V$. The result is

$$
\mathcal{N}(\boldsymbol{v})=\frac{1}{2 V+1} \sum_{m=0}^{V} \frac{(-1)^{m}(2 m) !(2 V-2 m) !}{2^{V} m !(V-m) !}=\frac{(2 V) !}{2^{V}(V+1) !} \frac{(-1)^{V}+1}{2}
$$


where the last equality can be found in [26]. The expression vanishes if $V$ is odd or equivalently if $n$ is even. With $V=n-1, n$ odd we obtain $K_{n, n-1}=1 / n$ ! and complete expansion of the form factor is $(n=2 k+1)$

$$
K(\tau, x)=\tau e^{-B \tau}+e^{-B \tau} \sum_{k=1}^{\infty} \frac{\tau^{4 k+1} B^{2 k}}{(2 k+1) !}=\frac{\sinh \left(B \tau^{2}\right)}{B \tau} \mathrm{e}^{-B \tau}
$$

in agreement with the RMT result (11).

\section{Conclusions}

This work is a continuation of recent developments in semiclassical periodic orbit expansions. These methods have been applied to several spectral and transport quantities in order to demonstrate the universality of quantum fluctuation statistics of chaotic systems. We extended these ideas to obtain a semiclassical expansion of the parametric spectral form factor $K(\tau, x)$ for small $\tau$ in agreement with RMT. For the GUE case we showed agreement for all orders, while for the GOE case we showed agreement for the first seven terms in the expansion. These terms can actually be obtained more quickly by the semiclassical method than by an expansion of the double integral for the GOE result.

The main input that is needed in addition to the semiclassical calculation of the spectral form factor $K(\tau)$ is the distribution of the parametric velocities of long orbits. This is the distribution of the derivatives of the actions of periodic orbits with respect to the parameter, which is commonly assumed to be Gaussian. In addition, when averaging over orbit pairs whose correlations are described by a particular structure, one has to take into account correlations between different parts of the same trajectory. These correlations are due to almost identical orbit stretches within the encounter regions.

The limitations of the semiclassical calculation are similar to that for the spectral form factor. One main open point concerns the region $\tau>1$. In this regime the random matrix expressions for $K(\tau, x)$ have a different functional form. So far it is not known how to extend the semiclassical approach to this regime except for a small region near $\tau=1[17,18]$.

\section{A Recurrence relations}

In this appendix we show that the quantities $S_{n}\left[f_{r}(\boldsymbol{v})\right]$, defined in (51) and (52), vanish for $r \leq n-1$. The function $f_{r}(\boldsymbol{v})$ are defined in terms of a restricted sum in which all summation indices are distinct. As a first step this sum is expressed by unrestricted sums. How to do this by a combinatorial sieving is discussed, for example, in section 4 of reference [27].

We first introduce some notation. A set partition $\boldsymbol{F}$ of the set of integers $\{1,2, \ldots r\}$ is a decomposition of this set into disjoint subsets $\left[F_{1}, \ldots, F_{\nu}\right]$. Then $\left|F_{1}\right|+\ldots+\left|F_{\nu}\right|=r$ where $\left|F_{i}\right|$ is the number of elements in the set $F_{i}$. Let us define a generalization of the Kronecker delta-function

$$
\delta_{\alpha_{1}, \ldots, \alpha_{r}}^{\boldsymbol{F}}= \begin{cases}1 & \text { if } \alpha_{i}=\alpha_{j} \text { for all } i \text { and } j \text { such that } i, j \in F_{k} \text { for some } k \\ 0 & \text { otherwise }\end{cases}
$$


Then

$$
\sum_{\substack{\alpha_{1}, \ldots, \alpha_{r} \\ \text { distinct }}}[\ldots]=\sum_{\boldsymbol{F}} \mu(\boldsymbol{F}) \sum_{\alpha_{1}, \ldots, \alpha_{r}} \delta_{\alpha_{1}, \ldots, \alpha_{r}}^{\boldsymbol{F}}[\ldots],
$$

where the first sum of the right-hand side runs over all set partitions of the set of $r$ integers, and the corresponding Möbius function is given by

$$
\mu(\boldsymbol{F})=\prod_{i=1}^{\nu}(-1)^{\left|F_{i}\right|-1}\left(\left|F_{i}\right|-1\right) !
$$

If we apply this to the functions $f_{r}(\boldsymbol{v})$ we obtain

$$
f_{r}(\boldsymbol{v})=\frac{(L-V-1) !}{(L-V-1+r) ! r !} \sum_{\boldsymbol{F}} \mu(\boldsymbol{F}) g_{\boldsymbol{F}}(\boldsymbol{v}),
$$

where

$$
g_{\boldsymbol{F}}(\boldsymbol{v})=\left(\sum_{k} v_{k}(k-1)^{\left|F_{1}\right|}\right) \times \ldots \times\left(\sum_{k} v_{k}(k-1)^{\left|F_{\nu}\right|}\right) .
$$

The first few functions $f_{r}(\boldsymbol{v})$ were given in equation (53).

The expansion of the form factor $K(\tau)$ was evaluated in $[9,10]$ by using recurrence relations for the number of structures $\mathcal{N}(\boldsymbol{v})$ corresponding to a vector $\boldsymbol{v}$. These recurrence relations were obtained by relating orbits with $L$ loops to orbits with $L-1$ loops by considering all possible ways of removing a loop (i.e. letting its size shrink to zero).

For systems without time reversal symmetry the relevant recurrence relation is

$$
v_{2} \tilde{N}(\boldsymbol{v})+\sum_{k \geq 2} v_{k+1}^{[k, 2 \rightarrow k+1]} k \tilde{N}\left(\boldsymbol{v}^{[k, 2 \rightarrow k+1]}\right)=0 .
$$

Here the vector $\boldsymbol{v}^{[k, 2 \rightarrow k+1]}$ is obtained from the vector $\boldsymbol{v}$ by decreasing the components $v_{k}$ and $v_{2}$ by one and increasing the component $v_{k+1}$ by one. Hence $L\left(\boldsymbol{v}^{[k, 2 \rightarrow k+1]}\right)=L(\boldsymbol{v})-k-2+(k+1)=$ $L(\boldsymbol{v})-1$ and $V\left(\boldsymbol{v}^{[k, 2 \rightarrow k+1]}\right)=V(\boldsymbol{v})-1$.

In order to obtain the coefficient of the form factor expansion one has to sum over the numbers $\mathcal{N}(\boldsymbol{v})$ for all vectors for which $L(\boldsymbol{v})-V(\boldsymbol{v})+1=n$. The recurrence relation may be used for this purpose, because one can show that for each $k$

$$
\sum_{\boldsymbol{v}}^{L-V+1=n} v_{k+1}^{[k, 2 \rightarrow k+1]} h\left(\boldsymbol{v}^{[k, 2 \rightarrow k+1]}\right)=\sum_{\boldsymbol{v}^{\prime}}^{L^{\prime}-V^{\prime}+1=n} v_{k+1}^{\prime} h\left(\boldsymbol{v}^{\prime}\right),
$$

where $h(\boldsymbol{v})$ is some function of $\boldsymbol{v}$. One condition is that $v_{1}=v_{1}^{[k, 2 \rightarrow k+1]}=0$, because the vectors describe encounter regions which contain at least two orbit stretches. Summing the recurrence relation (64) over $\boldsymbol{v}$ yields

$$
0=S_{n}\left[v_{2}+\sum_{k \geq 2} v_{k+1} k\right]=S_{n}[L-V]=(n-1) S_{n}[1] .
$$

This shows, for example, that all off-diagonal terms of the form factor $K(\tau, 0)$ vanish $[9,10]$. 
We want to show in the following that $S_{n}\left[g_{\boldsymbol{F}}(\boldsymbol{v})\right]=0$ if $r<n-1$. We consider first the case when the partition consists of only one subset $F_{1}$ with $\left|F_{1}\right|=r$. Then $g_{\boldsymbol{F}}(\boldsymbol{v})=g_{r}(\boldsymbol{v})$ where

$$
g_{r}(\boldsymbol{v})=\sum_{k} v_{k}(k-1)^{r} .
$$

We show that $S_{n}\left[g_{r}(\boldsymbol{v})\right]=0$ if $r<n-1$ by induction. The statement is true for $r=0$, because $S_{n}[1]=0$ by equation (66). Now we fix a value of $r<n-1$ and assume that the statement is true for all smaller values of $r$. From the definition (67) follows that

$$
g_{r}\left(\boldsymbol{v}^{[k, 2 \rightarrow k+1]}\right)=g_{r}(\boldsymbol{v})-h_{r}(k), \quad h_{r}(k)=(k-1)^{r}-k^{r}+1 .
$$

Points that will be important in the following are that $h_{r}(1)=0$ and that $h_{r}(k)$ is given by a finite power series in $k$ whose highest order term is $-r k^{r-1}$.

Multiplying equation (64) by $g_{r}(\boldsymbol{v})$ and using relation (68) we obtain

$$
0=v_{2} g_{r}(\boldsymbol{v}) \tilde{N}(\boldsymbol{v})+\sum_{k \geq 2} v_{k+1}^{\prime} k g_{r}\left(\boldsymbol{v}^{\prime}\right) \tilde{N}\left(\boldsymbol{v}^{\prime}\right)+\sum_{k \geq 2} v_{k+1}^{\prime} k h_{r}(k) \tilde{N}\left(\boldsymbol{v}^{\prime}\right)
$$

where $\boldsymbol{v}^{\prime}=\boldsymbol{v}^{[k, 2 \rightarrow k+1]}$. In the last sum we can start the sum at $k=1$, because $h_{r}(1)=0$, and then change the summation index $k \rightarrow k-1$. After summing over all vectors $\boldsymbol{v}$ we obtain

$$
\begin{aligned}
0 & =S_{n}\left[v_{2} g_{r}(\boldsymbol{v})+\sum_{k \geq 2} v_{k+1} k g_{r}(\boldsymbol{v})+\sum_{k \geq 2} v_{k}(k-1) h_{r}(k-1)\right. \\
& =S_{n}\left[(L-V) g_{r}(\boldsymbol{v})-\sum_{k \geq 2} v_{k} r(k-1)^{r}+\ldots\right] .
\end{aligned}
$$

In the second line we used that $v_{2}+\sum_{k \geq 2}^{\infty} v_{k+1} k=\sum_{l \geq 2} v_{l}(k-1)=L-V$, and we wrote only the highest order term of $h_{r}(k-1)$. The lower order terms, denoted by the dots, involve powers $(k-1)^{m}$ with $m<r$ and can be neglected due to our induction assumption. Hence we find that

$$
(n-r-1) S_{n}\left[g_{r}(\boldsymbol{v})\right]=0,
$$

so that indeed $S_{n}\left[g_{r}(\boldsymbol{v})\right]=0$ if $r<n-1$. The proof for general $g_{\boldsymbol{F}}(\boldsymbol{v})$ is very similar. We consider the general form

$$
g_{\boldsymbol{F}}(\boldsymbol{v})=\prod_{i=1}^{\nu} g_{\left|F_{i}\right|}(\boldsymbol{v}),
$$

and we use again induction to prove that $S_{n}\left[g_{\boldsymbol{F}}\right]=0$ if $r<n-1$. The statement is true for $r=0$, and we fix a value of $r$ and assume that it is true for all smaller values of $r$. In order to use the recurrence relation (64) we note that

$$
g_{\boldsymbol{F}}(\boldsymbol{v})=\prod_{i=1}^{\nu}\left(g_{\left|F_{i}\right|}\left(\boldsymbol{v}^{[k, 2 \rightarrow k+1]}\right)+h_{\left|F_{i}\right|}(k)\right) .
$$

We multiply equation (64) by $g_{\boldsymbol{F}}(\boldsymbol{v})$ and use relation (73) to obtain

$$
\begin{aligned}
0= & v_{2} g_{\boldsymbol{F}}(\boldsymbol{v}) \tilde{N}(\boldsymbol{v})+\sum_{k \geq 2} v_{k+1}^{\prime} k g_{\boldsymbol{F}}\left(\boldsymbol{v}^{\prime}\right) \tilde{N}\left(\boldsymbol{v}^{\prime}\right) \\
& +\sum_{k \geq 2} v_{k+1}^{\prime} k\left[\prod_{i=1}^{\nu}\left(g_{\left|F_{i}\right|}\left(\boldsymbol{v}^{\prime}\right)+h_{\left|F_{i}\right|}(k)\right)-\prod_{i=1}^{\nu} g_{\left|F_{i}\right|}\left(\boldsymbol{v}^{\prime}\right)\right] \tilde{N}\left(\boldsymbol{v}^{\prime}\right),
\end{aligned}
$$


where we added an additional term and subtracted it again. As before $\boldsymbol{v}^{\prime}=\boldsymbol{v}^{[k, 2 \rightarrow k+1]}$. In the second sum we can start the sum at $k=1$, because $h_{i}(1)=0$ for all $i$, and then change the summation index $k \rightarrow k-1$. After summing over all vectors $\boldsymbol{v}$ we obtain

$$
\begin{aligned}
0 & =S_{n}\left[v_{2} g_{\boldsymbol{F}}(\boldsymbol{v})+\sum_{k \geq 2} v_{k+1} k g_{\boldsymbol{F}}(\boldsymbol{v})+\sum_{k \geq 2} v_{k}(k-1)\left[\prod_{i=1}^{\nu}\left(g_{\left|F_{i}\right|}(\boldsymbol{v})+h_{\left|F_{i}\right|}(k-1)\right)-\prod_{i=1}^{\nu} g_{\left|F_{i}\right|}(\boldsymbol{v})\right]\right. \\
& =S_{n}\left[(L-V) g_{\boldsymbol{F}}(\boldsymbol{v})+\sum_{k \geq 2} v_{k}(k-1) \sum_{j=1}^{\nu}\left(-\left|F_{j}\right|(k-1)^{\left|F_{j}\right|-1}\right) \prod_{i \neq j} g_{\left|F_{i}\right|}(\boldsymbol{v})+\ldots\right] .
\end{aligned}
$$

In the step from the first to the second line we expanded the first product, inserted the power series for the functions $h_{\left|F_{i}\right|}(k-1)$ and wrote only those terms that do not vanish due to the induction assumption. We obtain further

$$
\begin{aligned}
0 & =S_{n}\left[(L-V) g_{\boldsymbol{F}}(\boldsymbol{v})-\sum_{j=1}^{\nu}\left|F_{j}\right| g_{\left|F_{i}\right|}(\boldsymbol{v}) \prod_{i \neq j} g_{\left|F_{i}\right|}(\boldsymbol{v})+\ldots\right] \\
& =(n-1-r) S_{n}\left[g_{\boldsymbol{F}}(\boldsymbol{v})\right]
\end{aligned}
$$

which concludes the proof that $S_{n}\left[g_{\boldsymbol{F}}(\boldsymbol{v})\right]=0$ for $r<n-1$.

\section{Acknowledgements}

The authors would like to thank EPSRC for financial support.

\section{References}

[1] O. Bohigas, M. J. Giannoni, and C. Schmit. Characterization of chaotic quantum spectra and universality of level fluctuation laws. Phys. Rev. Lett., 52:1-4, 1984.

[2] B. D. Simons and B. L. Altshuler. Universal velocity correlations in disordered and chaotic systems. Phys. Rev. Lett., 70:4063-4066, 1993.

[3] A. Szafer and B. L. Altshuler. Universal correlation in the spectra of disordered systems with an aharonov-bohm flux line. Phys. Rev. Lett., 70:587-590, 1993.

[4] T. Guhr, A. Müller-Groeling, and Hans A. Weidenmüller. Random matrix theories in quantum physics: Common concepts. Phys. Rep., 299:189-425, 1998.

[5] J. H. Hannay and A. M. Ozorio de Almeida. Periodic orbits and a correlation function for the semiclassical density of states. J. Phys. A, 17:3429-3440, 1984.

[6] M. V. Berry. Semiclassical theory of spectral rigidity. Proc. R. Soc. Lond. A, 400:229-251, 1985.

[7] M. Sieber and K. Richter. Correlations between periodic orbits and their rôle in spectral statistics. Physica Scripta, T90:128-133, 2001. 
[8] M. Sieber. Leading off-diagonal approximation for the spectral form factor for uniformly hyperbolic systems. J. Phys. A, 35:L613-L619, 2002.

[9] S. Müller, S. Heusler, P. Braun, F. Haake, and A. Altland. Semiclassical foundation of universality in quantum chaos. Phys. Rev. Lett., 93:014103, 2004.

[10] S. Müller, S. Heusler, P. Braun, F. Haake, and A. Altland. Periodic-orbit theory of universality in quantum chaos. Phys. Rev. E., 72:046207, 2005.

[11] S. Müller. Periodic-Orbit Approach to Universality in Quantum Chaos. PhD thesis, Universität Duisburg-Essen, 2005. nlin.CD/0512058.

[12] K. Richter and M. Sieber. Semiclassical theory of chaotic quantum transport. Phys. Rev. Lett., 89:206801, 2002.

[13] S. Heusler, S. Müller, P. Braun, and F. Haake. Semiclassical theory of chaotic conductors. Phys. Rev. Lett., 96:066804, 2006.

[14] P. Braun, S. Heusler, S. Müller, and F. Haake. Semiclassical prediction for shot noise in chaotic cavities. J. Phys. A, 39:L159-L165, 2006.

[15] K. Saito and T. Nagao. Spectral form factor for chaotic dynamics in a weak magnetic field. Phys. Lett. A, 352:380-385, 2006.

[16] J. Goldberg, U. Smilansky, M. V. Berry, W. Schweizer, G. Wunner, and G. Zeller. The parametric number variance. Nonlinearity, 4:1-14, 1991.

[17] E. B. Bogomolny and J. P. Keating. Gutzwiller's trace formula and spectral statistics: Beyond the diagonal approximation. Phys. Rev. Lett., 77:1472-1475, 1996.

[18] A. M. Ozorio de Almeida, C. H. Lewenkopf, and E. R. Mucciolo. Semiclassical limit of universal parametric density correlations. Phys. Rev. E, 58:5693-5703, 1998.

[19] M. Sieber. Spectral statistics in chaotic systems with a point interaction. J. Phys. A, 33:6263-6278, 2000.

[20] T. Nagao, P. Braun, S. Müller, K. Saito, S. Heusler, and F. Haake. Semiclassical theory for parametric correlations of energy levels. nlin.CD/0607070, 2006.

[21] P. Leboeuf and M. Sieber. Universality in quantum parametric correlations. Phys. Rev. E, 60:3969-3972, 1999.

[22] B. D. Simons and B. L. Altshuler. Universalities in the spectra of disordered and chaotic systems. Phys. Rev. B, 48:5422-5438, 1993.

[23] M. C. Gutzwiller. Periodic orbits and classical quantization conditions. J. Math. Phys., 12:343-358, 1971.

[24] B. Eckhardt, S. Fishman, J. Keating, O. Agam, J. Main, and K. Müller. Approach to ergodicity in quantum wave functions. Phys. Rev. E, 52:5893-5903, 1995.

[25] J. Müller. On a mysterious partition identity. Preprint, 2003. 
[26] A. P. Prudnikov, Yu. A. Brychkov, and O. I. Marichev. Integrals and Series, Volume 1. Gordon and Breach Science Publishers, New York, 1988. Second printing with corrections.

[27] Zeev Rudnik and Peter Sarnak. Zeros of principle $l$-functions and random matrix theory. Duke Math. J., 81:269-322, 1996. 